Network Working Group

Request for Comments: 2460

Obsoletes: 1883

Category: Standards Track
S. Deering

Cisco

R. Hinden

Nokia

December 1998

\title{
Internet Protocol, Version 6 (IPv6) \\ Specification
}

\section{Status of this Memo}

This document specifies an Internet standards track protocol for the Internet community, and requests discussion and suggestions for improvements. Please refer to the current edition of the "Internet Official Protocol Standards" (STD 1) for the standardization state and status of this protocol. Distribution of this memo is unlimited.

Copyright Notice

Copyright (C) The Internet Society (1998). All Rights Reserved.

Abstract

This document specifies version 6 of the Internet Protocol (IPv6), also sometimes referred to as IP Next Generation or IPng.

Table of Contents

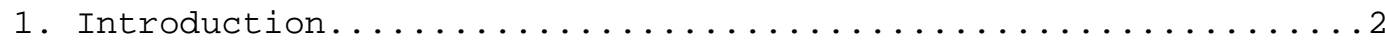

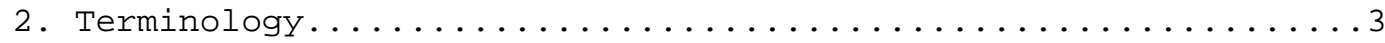

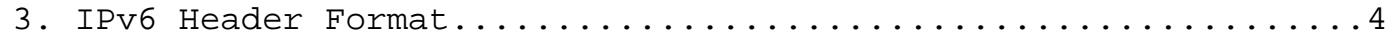

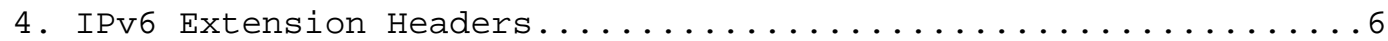

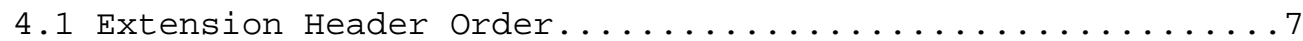

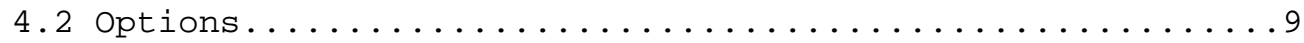

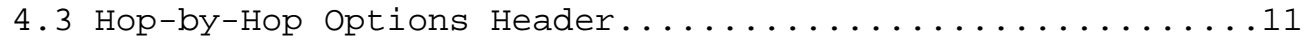

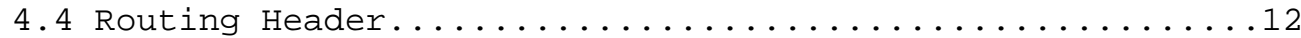

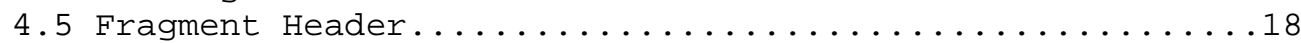

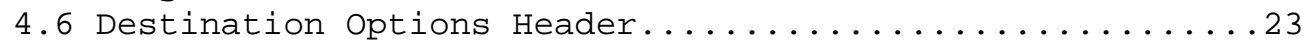

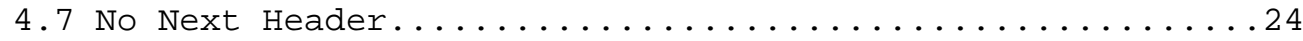

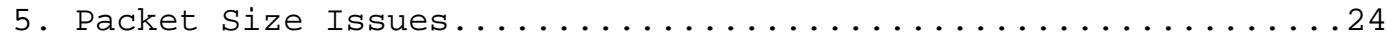

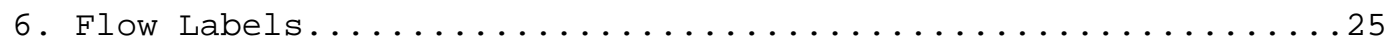

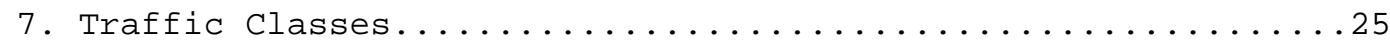

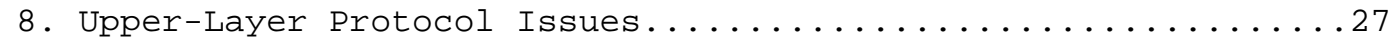

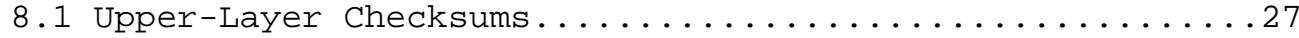

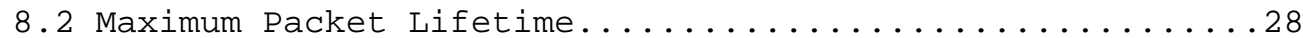

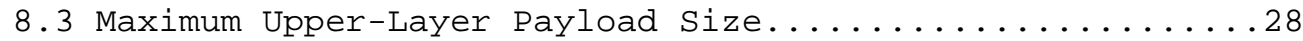

8.4 Responding to Packets Carrying Routing Headers........29 
Appendix A. Semantics and Usage of the Flow Label Field.......30

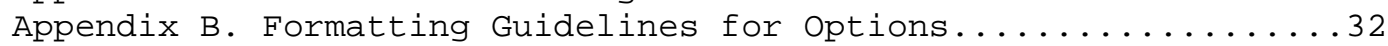

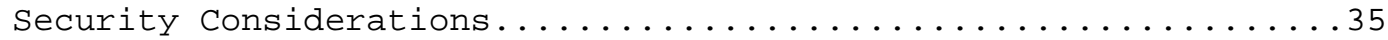

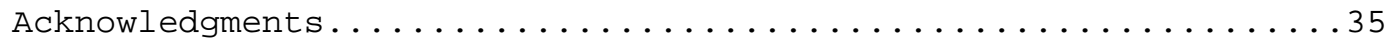

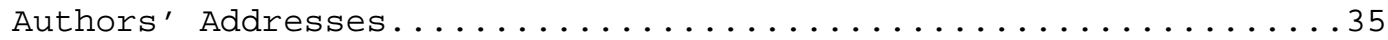

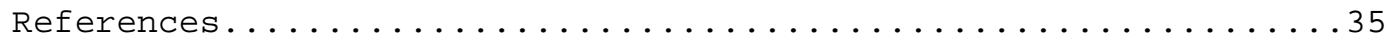

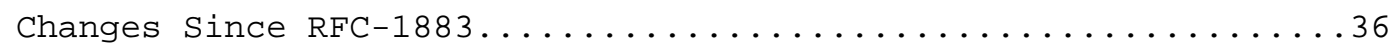

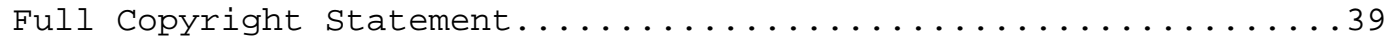

\section{Introduction}

IP version 6 (IPv6) is a new version of the Internet Protocol, designed as the successor to IP version 4 (IPV4) [RFC-791]. The changes from IPv4 to IPv6 fall primarily into the following categories:

- Expanded Addressing Capabilities

IPv6 increases the IP address size from 32 bits to 128 bits, to support more levels of addressing hierarchy, a much greater number of addressable nodes, and simpler auto-configuration of addresses. The scalability of multicast routing is improved by adding a "scope" field to multicast addresses. And a new type of address called an "anycast address" is defined, used to send a packet to any one of a group of nodes.

- Header Format Simplification

Some IPv4 header fields have been dropped or made optional, to reduce the common-case processing cost of packet handling and to limit the bandwidth cost of the IPv6 header.

- Improved Support for Extensions and Options

Changes in the way IP header options are encoded allows for more efficient forwarding, less stringent limits on the length of options, and greater flexibility for introducing new options in the future.

- Flow Labeling Capability

A new capability is added to enable the labeling of packets belonging to particular traffic "flows" for which the sender requests special handling, such as non-default quality of service or "real-time" service. 
- Authentication and Privacy Capabilities

Extensions to support authentication, data integrity, and (optional) data confidentiality are specified for IPv6.

This document specifies the basic IPv6 header and the initiallydefined IPv6 extension headers and options. It also discusses packet size issues, the semantics of flow labels and traffic classes, and the effects of IPv6 on upper-layer protocols. The format and semantics of IPV6 addresses are specified separately in [ADDRARCH]. The IPV6 version of ICMP, which all IPV6 implementations are required to include, is specified in [ICMPv6].

2. Terminology

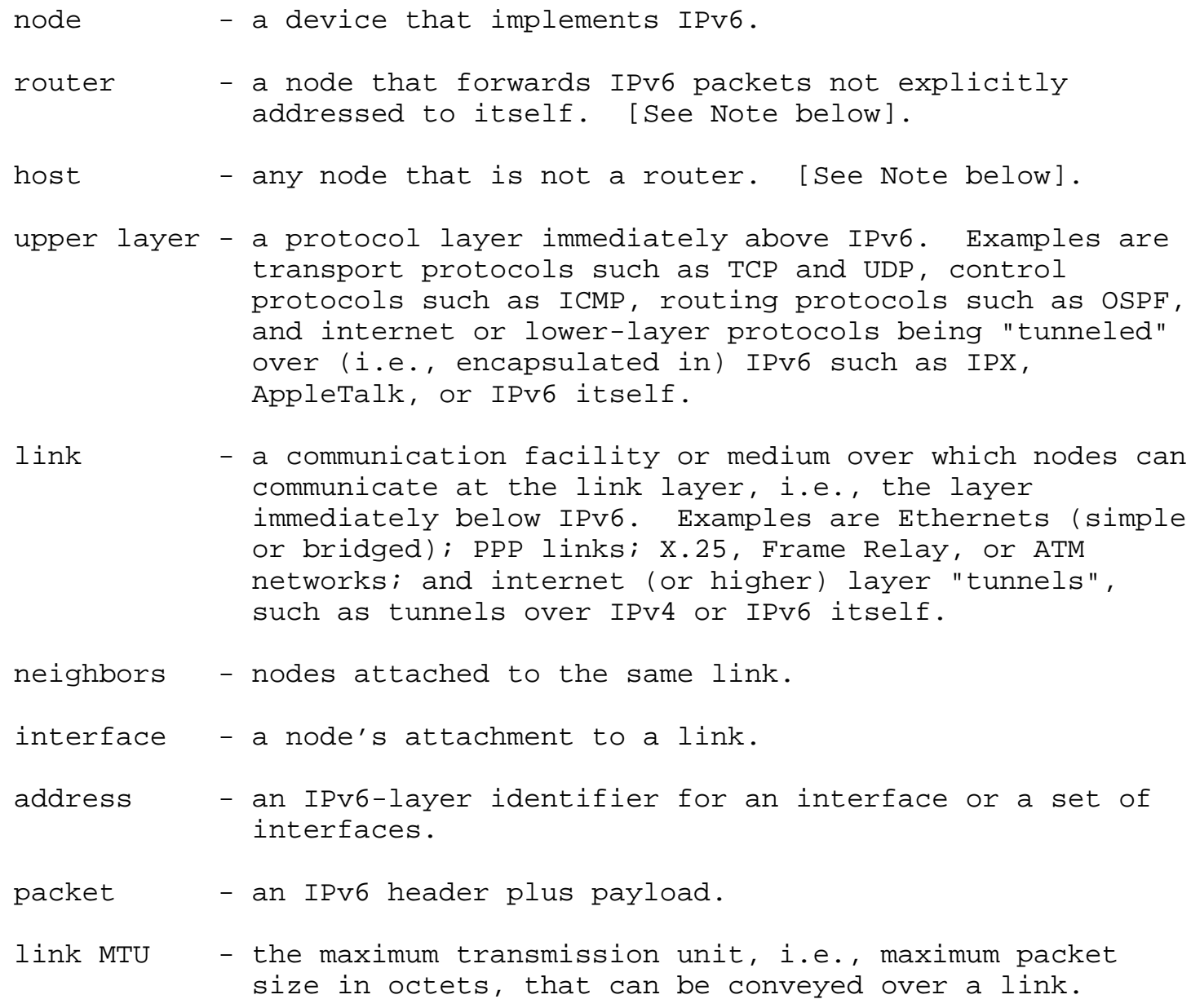


path MTU - the minimum link MTU of all the links in a path between a source node and a destination node.

Note: it is possible, though unusual, for a device with multiple interfaces to be configured to forward non-self-destined packets arriving from some set (fewer than all) of its interfaces, and to discard non-self-destined packets arriving from its other interfaces. Such a device must obey the protocol requirements for routers when receiving packets from, and interacting with neighbors over, the former (forwarding) interfaces. It must obey the protocol requirements for hosts when receiving packets from, and interacting with neighbors over, the latter (non-forwarding) interfaces.

3. IPv6 Header Format

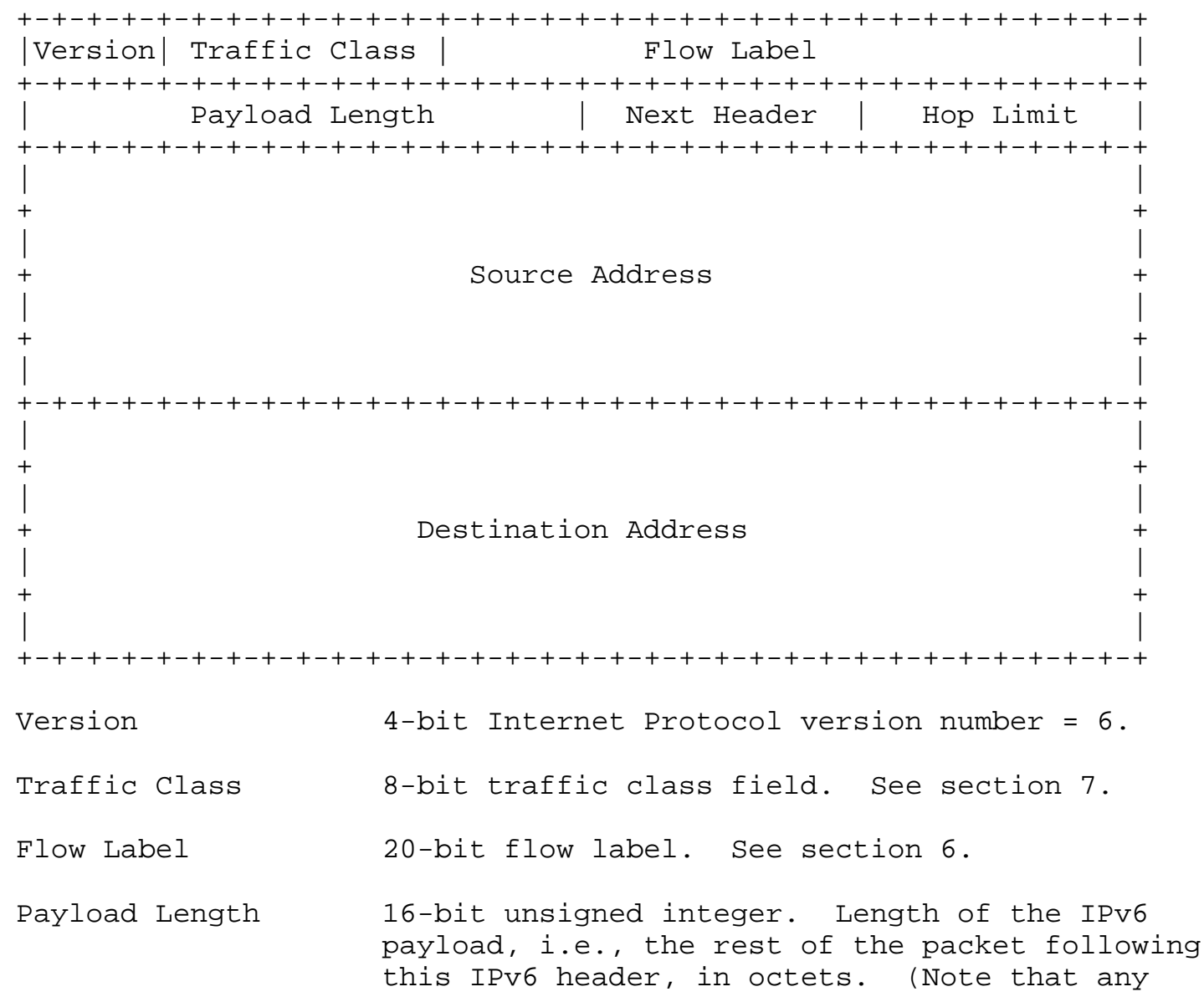


Next Header

Hop Limit

Source Address

Destination Address extension headers [section 4] present are considered part of the payload, i.e., included in the length count.)

8-bit selector. Identifies the type of header immediately following the IPv6 header. Uses the same values as the IPv4 Protocol field [RFC-1700 et seq.].

8-bit unsigned integer. Decremented by 1 by each node that forwards the packet. The packet is discarded if Hop Limit is decremented to zero.

128-bit address of the originator of the packet. See [ADDRARCH].

128-bit address of the intended recipient of the packet (possibly not the ultimate recipient, if a Routing header is present). See [ADDRARCH] and section 4.4 . 


\section{IPv6 Extension Headers}

In IPv6, optional internet-layer information is encoded in separate headers that may be placed between the IPv6 header and the upperlayer header in a packet. There are a small number of such extension headers, each identified by a distinct Next Header value. As illustrated in these examples, an IPv6 packet may carry zero, one, or more extension headers, each identified by the Next Header field of the preceding header:
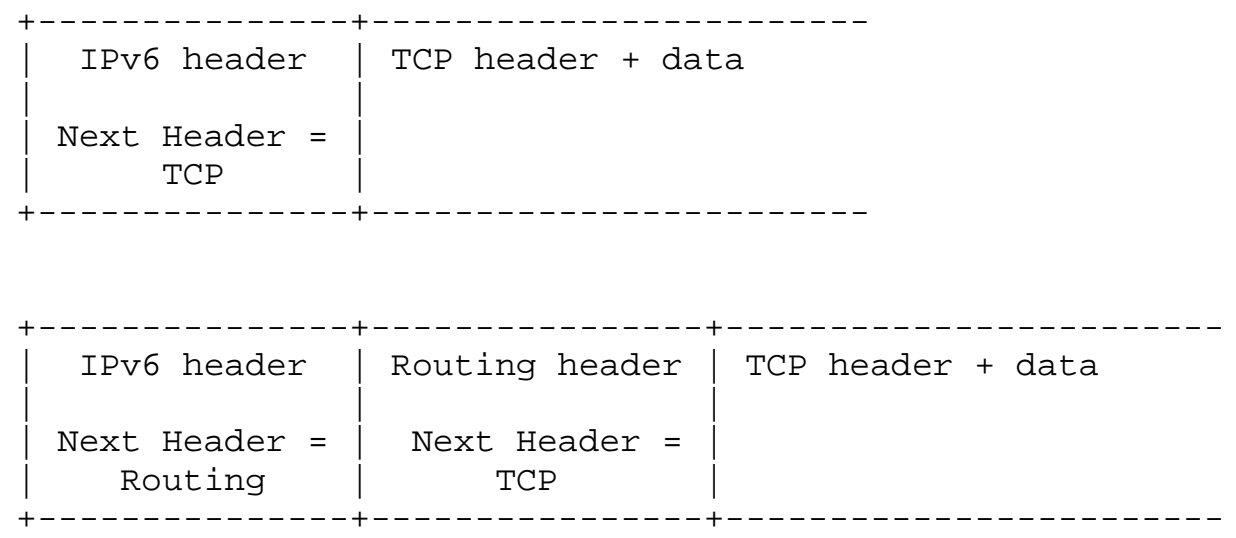

\begin{tabular}{|c|c|c|c} 
IPv6 header & Routing header & Fragment header & $\begin{array}{c}\text { fragment of TCP } \\
\text { header }+ \text { data }\end{array}$ \\
Routing & $\begin{array}{c}\text { Next Header }= \\
\text { Fragment }\end{array}$ & Next Header $=$ & TCP
\end{tabular}

With one exception, extension headers are not examined or processed by any node along a packet's delivery path, until the packet reaches the node (or each of the set of nodes, in the case of multicast) identified in the Destination Address field of the IPv6 header. There, normal demultiplexing on the Next Header field of the IPv6 header invokes the module to process the first extension header, or the upper-layer header if no extension header is present. The contents and semantics of each extension header determine whether or not to proceed to the next header. Therefore, extension headers must be processed strictly in the order they appear in the packet; a receiver must not, for example, scan through a packet looking for a particular kind of extension header and process that header prior to processing all preceding ones. 
The exception referred to in the preceding paragraph is the Hop-byHop Options header, which carries information that must be examined and processed by every node along a packet's delivery path, including the source and destination nodes. The Hop-by-Hop Options header, when present, must immediately follow the IPv6 header. Its presence is indicated by the value zero in the Next Header field of the IPv6 header.

If, as a result of processing a header, a node is required to proceed to the next header but the Next Header value in the current header is unrecognized by the node, it should discard the packet and send an ICMP Parameter Problem message to the source of the packet, with an ICMP Code value of 1 ("unrecognized Next Header type encountered") and the ICMP Pointer field containing the offset of the unrecognized value within the original packet. The same action should be taken if a node encounters a Next Header value of zero in any header other than an IPv6 header.

Each extension header is an integer multiple of 8 octets long, in order to retain 8-octet alignment for subsequent headers. Multioctet fields within each extension header are aligned on their natural boundaries, i.e., fields of width $n$ octets are placed at an integer multiple of $n$ octets from the start of the header, for $n=1$, 2,4 , or 8 .

A full implementation of IPv6 includes implementation of the following extension headers:

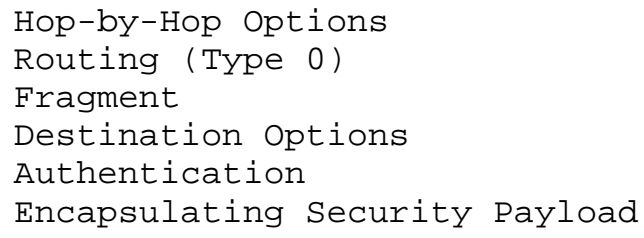

The first four are specified in this document; the last two are specified in [RFC-2402] and [RFC-2406], respectively.

\subsection{Extension Header Order}

When more than one extension header is used in the same packet, it is recommended that those headers appear in the following order:

IPv6 header

Hop-by-Hop Options header

Destination Options header (note 1)

Routing header

Fragment header 


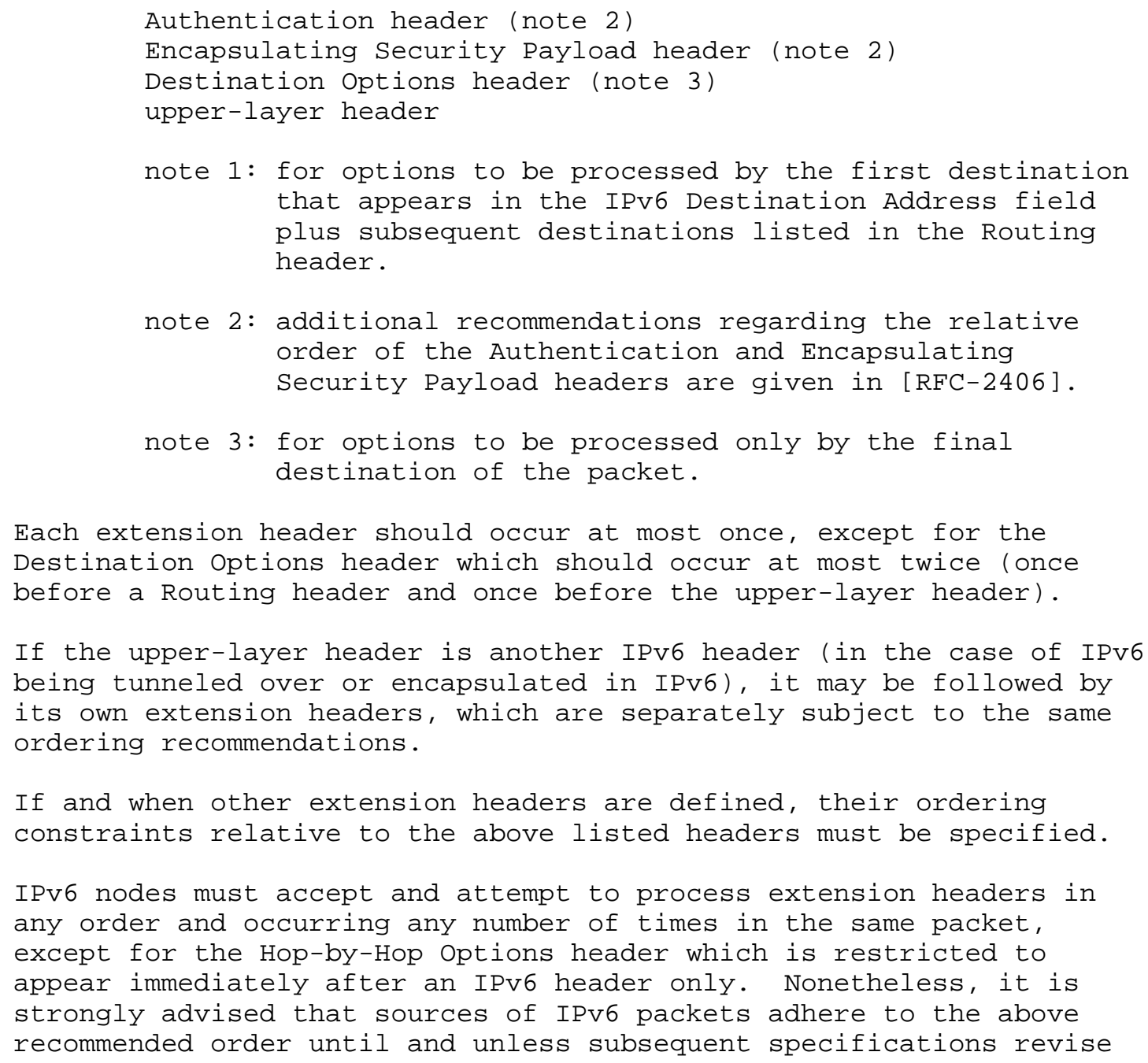




\subsection{Options}

Two of the currently-defined extension headers -- the Hop-by-Hop Options header and the Destination Options header -- carry a variable number of type-length-value (TLV) encoded "options", of the following format :

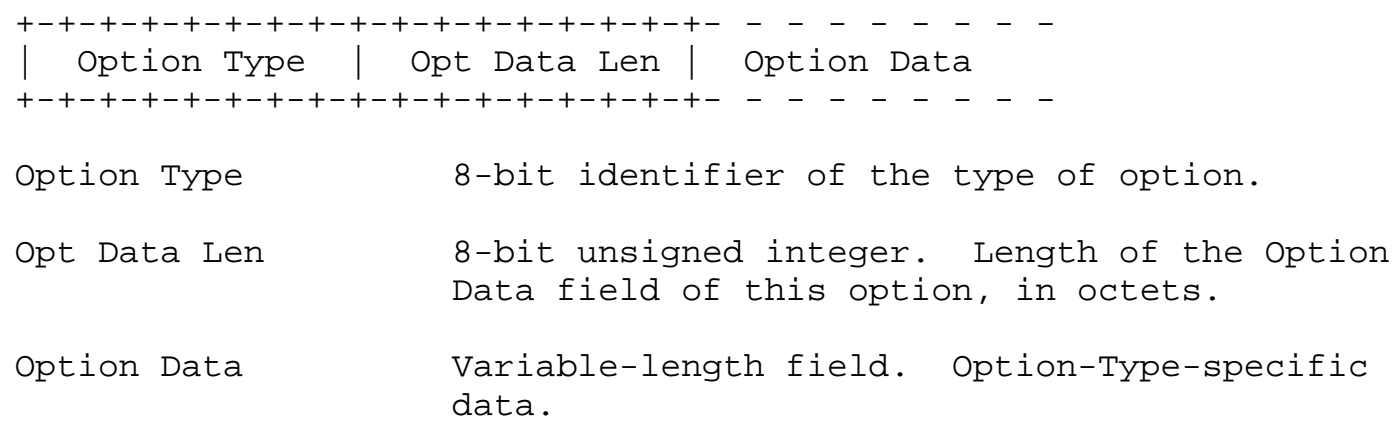

The sequence of options within a header must be processed strictly in the order they appear in the header; a receiver must not, for example, scan through the header looking for a particular kind of option and process that option prior to processing all preceding ones.

The Option Type identifiers are internally encoded such that their highest-order two bits specify the action that must be taken if the processing IPv6 node does not recognize the option Type:

00 - skip over this option and continue processing the header.

01 - discard the packet.

10 - discard the packet and, regardless of whether or not the packet's Destination Address was a multicast address, send an ICMP Parameter Problem, Code 2, message to the packet's Source Address, pointing to the unrecognized Option Type.

11 - discard the packet and, only if the packet's Destination Address was not a multicast address, send an ICMP Parameter Problem, Code 2, message to the packet's Source Address, pointing to the unrecognized Option Type.

The third-highest-order bit of the Option Type specifies whether or not the Option Data of that option can change en-route to the packet's final destination. When an Authentication header is present 
in the packet, for any option whose data may change en-route, its entire Option Data field must be treated as zero-valued octets when computing or verifying the packet's authenticating value.

$$
\begin{aligned}
& 0 \text { - Option Data does not change en-route } \\
& 1 \text { - Option Data may change en-route }
\end{aligned}
$$

The three high-order bits described above are to be treated as part of the Option Type, not independent of the Option Type. That is, a particular option is identified by a full 8-bit Option Type, not just the low-order 5 bits of an Option Type.

The same Option Type numbering space is used for both the Hop-by-Hop Options header and the Destination Options header. However, the specification of a particular option may restrict its use to only one of those two headers.

Individual options may have specific alignment requirements, to ensure that multi-octet values within Option Data fields fall on natural boundaries. The alignment requirement of an option is specified using the notation $x+y$, meaning the Option Type must appear at an integer multiple of $x$ octets from the start of the header, plus y octets. For example:

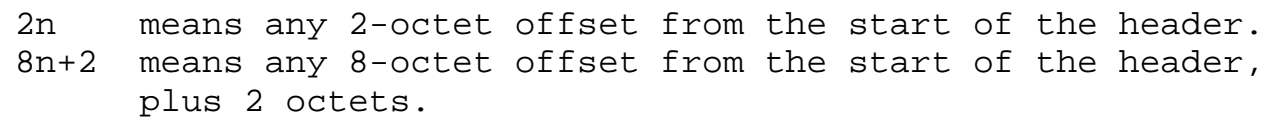

There are two padding options which are used when necessary to align subsequent options and to pad out the containing header to a multiple of 8 octets in length. These padding options must be recognized by all IPv6 implementations:

Pad1 option (alignment requirement: none)

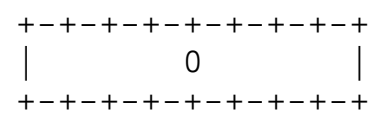

NOTE! the format of the Padl option is a special case -- it does not have length and value fields.

The Padl option is used to insert one octet of padding into the options area of a header. If more than one octet of padding is required, the PadN option, described next, should be used, rather than multiple Padl options. 
PadN option (alignment requirement: none)

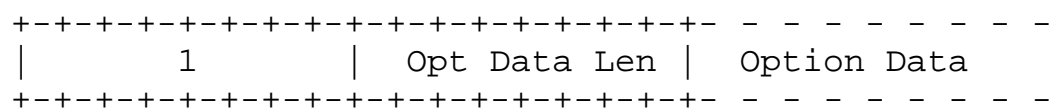

The PadN option is used to insert two or more octets of padding into the Options area of a header. For $N$ octets of padding, the Opt Data Len field contains the value $\mathrm{N}-2$, and the option Data consists of $\mathrm{N}-2$ zero-valued octets.

Appendix B contains formatting guidelines for designing new options.

\subsection{Hop-by-Hop Options Header}

The Hop-by-Hop Options header is used to carry optional information that must be examined by every node along a packet's delivery path. The Hop-by-Hop Options header is identified by a Next Header value of 0 in the IPv6 header, and has the following format:

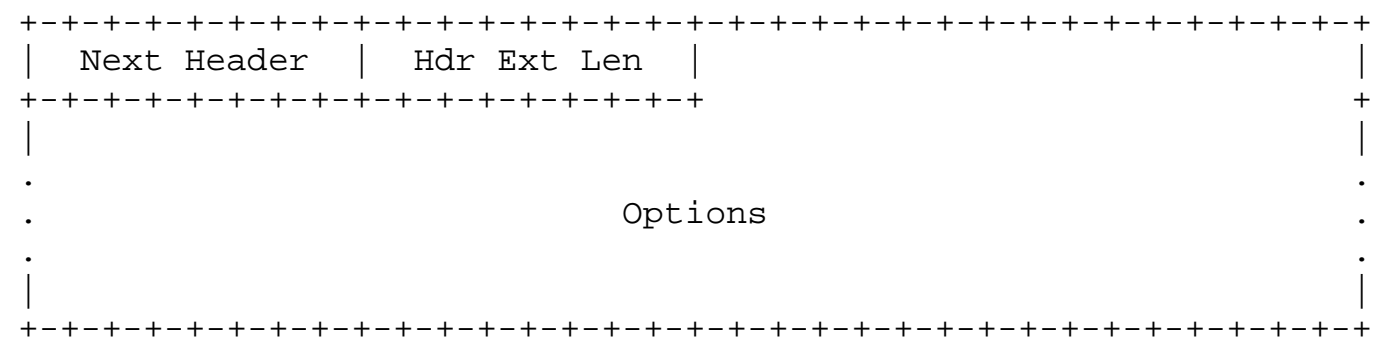

Next Header

8-bit selector. Identifies the type of header immediately following the Hop-by-Hop options header. Uses the same values as the IPv4 Protocol field [RFC-1700 et seq.].

Hdr Ext Len 8-bit unsigned integer. Length of the Hop-byHop Options header in 8-octet units, not including the first 8 octets.

Options

Variable-length field, of length such that the complete Hop-by-Hop Options header is an integer multiple of 8 octets long. Contains one or more TLV-encoded options, as described in section 4.2 .

The only hop-by-hop options defined in this document are the Padl and PadN options specified in section 4.2 . 


\subsection{Routing Header}

The Routing header is used by an IPv6 source to list one or more intermediate nodes to be "visited" on the way to a packet's destination. This function is very similar to IPv4's Loose source and Record Route option. The Routing header is identified by a Next Header value of 43 in the immediately preceding header, and has the following format:

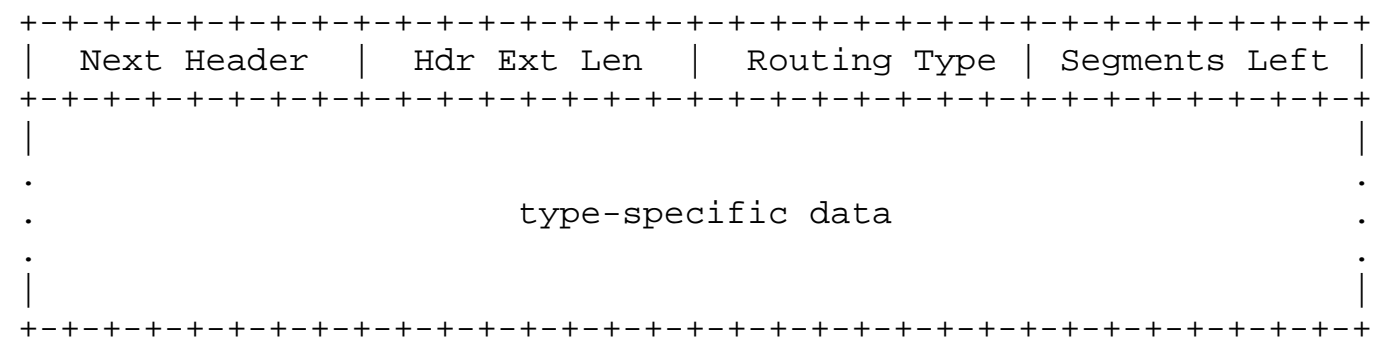

Next Header

8-bit selector. Identifies the type of header immediately following the Routing header. Uses the same values as the IPv4 Protocol field [RFC-1700 et seq.].

Hdr Ext Len

8-bit unsigned integer. Length of the Routing header in 8-octet units, not including the first 8 octets.

Routing Type

8-bit identifier of a particular Routing header variant.

Segments Left

8-bit unsigned integer. Number of route segments remaining, i.e., number of explicitly listed intermediate nodes still to be visited before reaching the final destination.

type-specific data Variable-length field, of format determined by the Routing Type, and of length such that the complete Routing header is an integer multiple of 8 octets long.

If, while processing a received packet, a node encounters a Routing header with an unrecognized Routing Type value, the required behavior of the node depends on the value of the segments Left field, as follows: 
If Segments Left is zero, the node must ignore the Routing header and proceed to process the next header in the packet, whose type is identified by the Next Header field in the Routing header.

If Segments Left is non-zero, the node must discard the packet and send an ICMP Parameter Problem, Code 0, message to the packet's Source Address, pointing to the unrecognized Routing Type.

If, after processing a Routing header of a received packet, an intermediate node determines that the packet is to be forwarded onto a link whose link MTU is less than the size of the packet, the node must discard the packet and send an ICMP Packet Too Big message to the packet's source Address. 
The Type 0 Routing header has the following format:

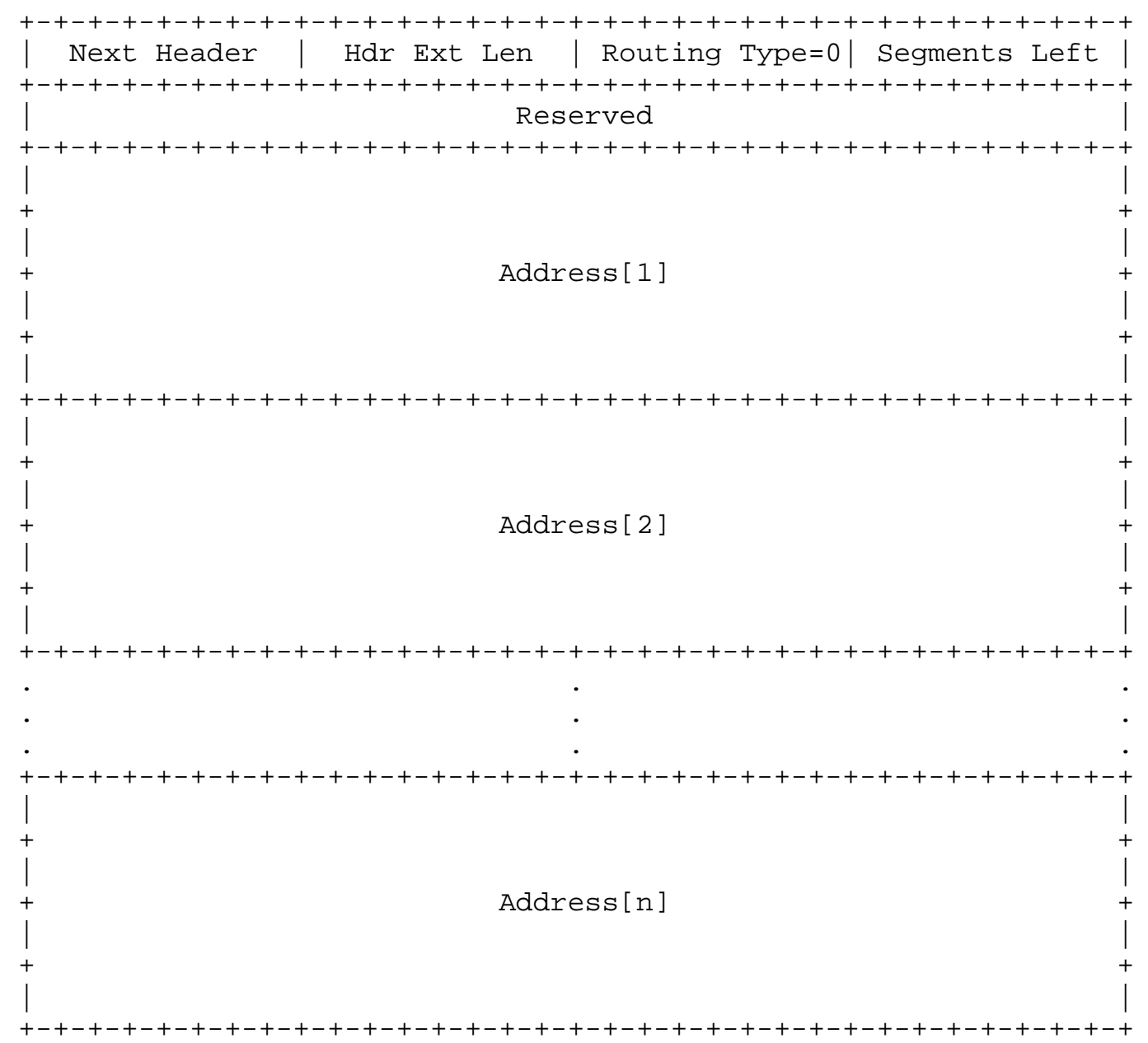

Next Header

8-bit selector. Identifies the type of header immediately following the Routing header. Uses the same values as the IPv4 Protocol field [RFC-1700 et seq.].

Hdr Ext Len 8-bit unsigned integer. Length of the Routing header in 8-octet units, not including the first 8 octets. For the Type 0 Routing header, Hdr Ext Len is equal to two times the number of addresses in the header.

Routing Type

0 . 


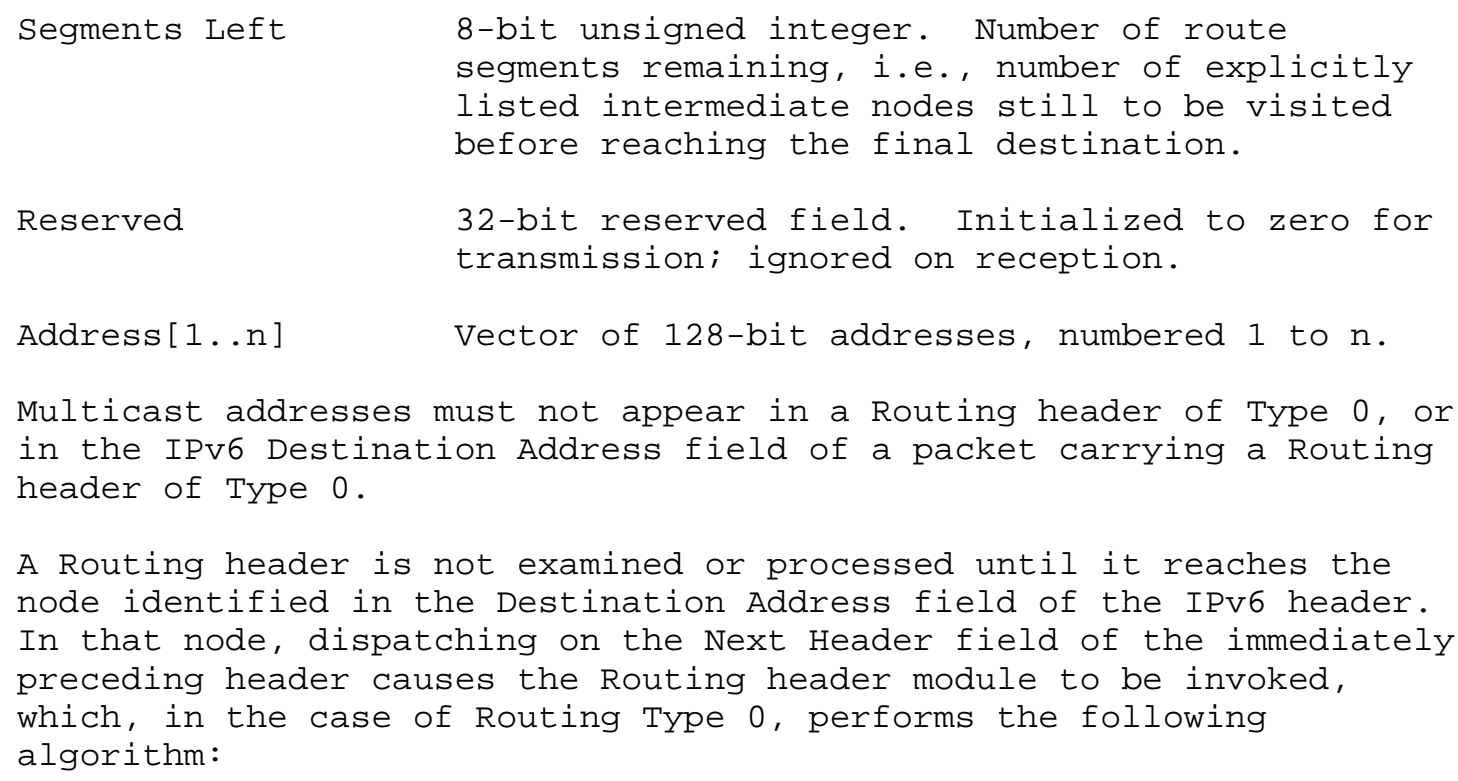




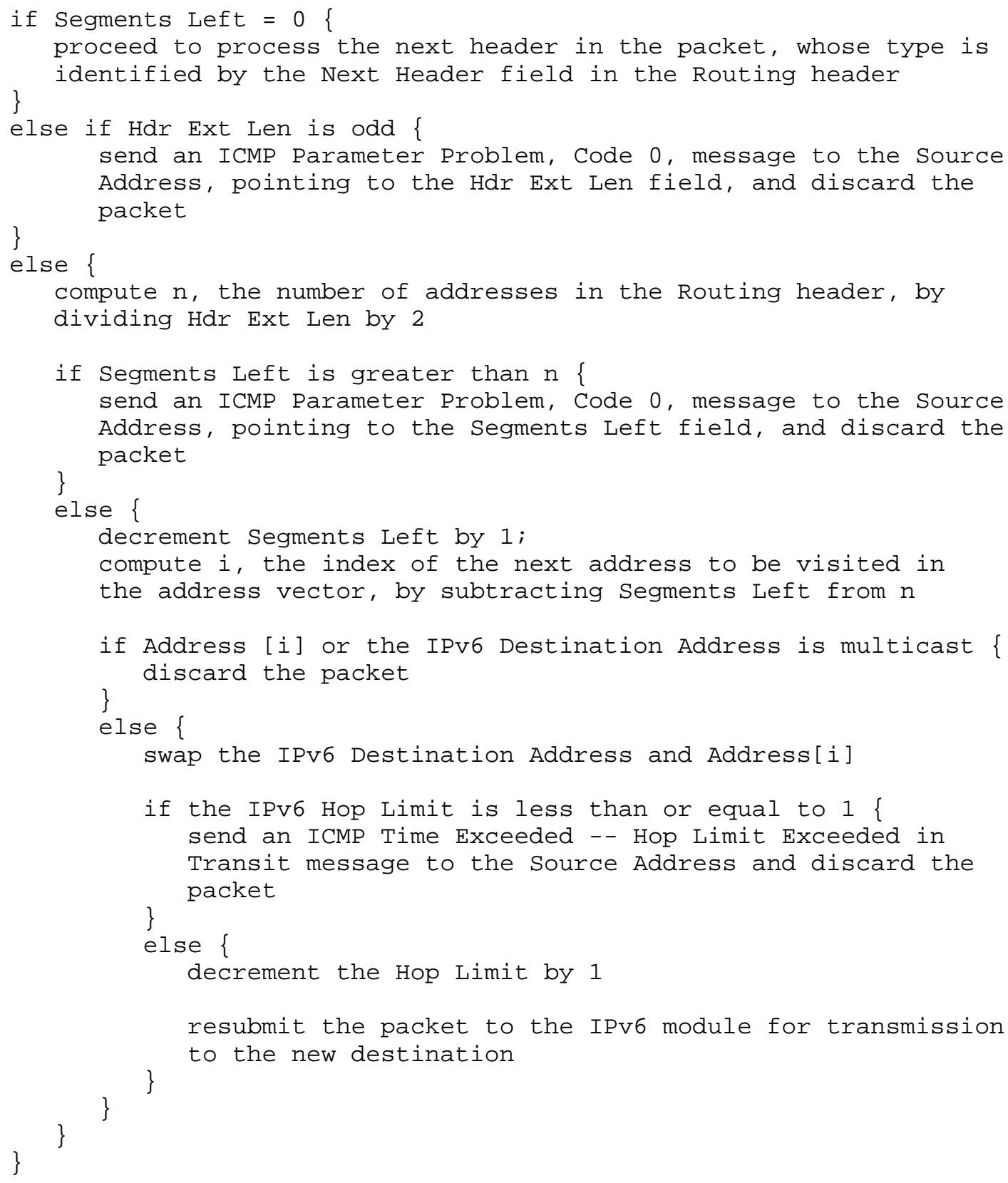


As an example of the effects of the above algorithm, consider the case of a source node $S$ sending a packet to destination node $D$, using a Routing header to cause the packet to be routed via intermediate nodes I1, I2, and I3. The values of the relevant IPV6 header and Routing header fields on each segment of the delivery path would be as follows:

As the packet travels from $S$ to II:

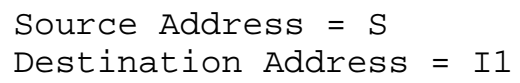

As the packet travels from I1 to I2:

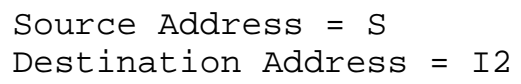

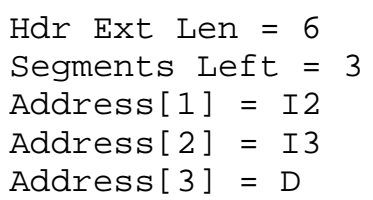

Hdr Ext Len $=6$ Segments Left $=2$ Address [1] = I1 Address [2] = I3 Address [3] = D

Hdr Ext Len $=6$ Segments Left $=1$ Address [1] = I1 Address [2] = I2 Address [3] = D

Hdr Ext Len $=6$ Segments Left $=0$ Address [1] = I1 Address [2] = I2 Address [3] = I3 


\subsection{Fragment Header}

The Fragment header is used by an IPv6 source to send a packet larger than would fit in the path MTU to its destination. (Note: unlike IPv4, fragmentation in IPv6 is performed only by source nodes, not by routers along a packet's delivery path -- see section 5.) The Fragment header is identified by a Next Header value of 44 in the immediately preceding header, and has the following format:

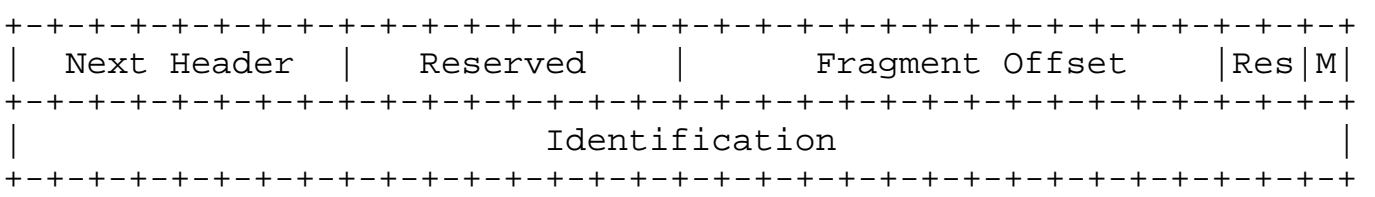

Next Header

8-bit selector. Identifies the initial header type of the Fragmentable Part of the original packet (defined below). Uses the same values as the IPv4 Protocol field [RFC-1700 et seq.].

Reserved

8-bit reserved field. Initialized to zero for transmission; ignored on reception.

Fragment Offset

13-bit unsigned integer. The offset, in 8-octet units, of the data following this header, relative to the start of the Fragmentable Part of the original packet.

Res 2-bit reserved field. Initialized to zero for transmission; ignored on reception.

M flag $1=$ more fragments; 0 = last fragment.

Identification 32 bits. See description below.

In order to send a packet that is too large to fit in the MTU of the path to its destination, a source node may divide the packet into fragments and send each fragment as a separate packet, to be reassembled at the receiver.

For every packet that is to be fragmented, the source node generates an Identification value. The Identification must be different than that of any other fragmented packet sent recently* with the same Source Address and Destination Address. If a Routing header is present, the Destination Address of concern is that of the final destination. 
* "recently" means within the maximum likely lifetime of a packet, including transit time from source to destination and time spent awaiting reassembly with other fragments of the same packet. However, it is not required that a source node know the maximum packet lifetime. Rather, it is assumed that the requirement can be met by maintaining the Identification value as a simple, 32bit, "wrap-around" counter, incremented each time a packet must be fragmented. It is an implementation choice whether to maintain a single counter for the node or multiple counters, e.g., one for each of the node's possible source addresses, or one for each active (source address, destination address) combination.

The initial, large, unfragmented packet is referred to as the "original packet", and it is considered to consist of two parts, as illustrated:

original packet:

\begin{tabular}{|c|c|}
\hline $\begin{array}{c}\text { Unfragmentable } \\
\text { Part }\end{array}$ & $\begin{array}{c}\text { Fragmentable } \\
\text { Part }\end{array}$ \\
\hline
\end{tabular}

The Unfragmentable Part consists of the IPv6 header plus any extension headers that must be processed by nodes en route to the destination, that is, all headers up to and including the Routing header if present, else the Hop-by-Hop Options header if present, else no extension headers.

The Fragmentable Part consists of the rest of the packet, that is, any extension headers that need be processed only by the final destination node(s), plus the upper-layer header and data.

The Fragmentable Part of the original packet is divided into fragments, each, except possibly the last ("rightmost") one, being an integer multiple of 8 octets long. The fragments are transmitted in separate "fragment packets" as illustrated:

original packet:

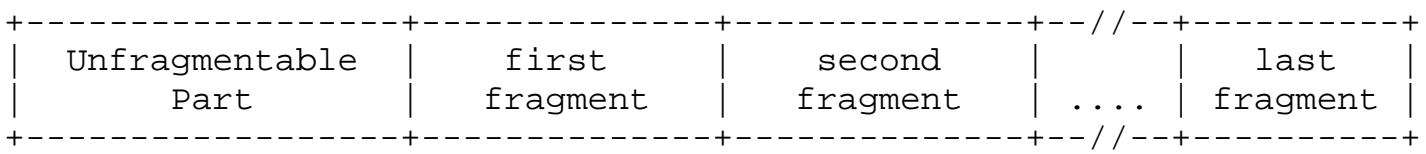


fragment packets:

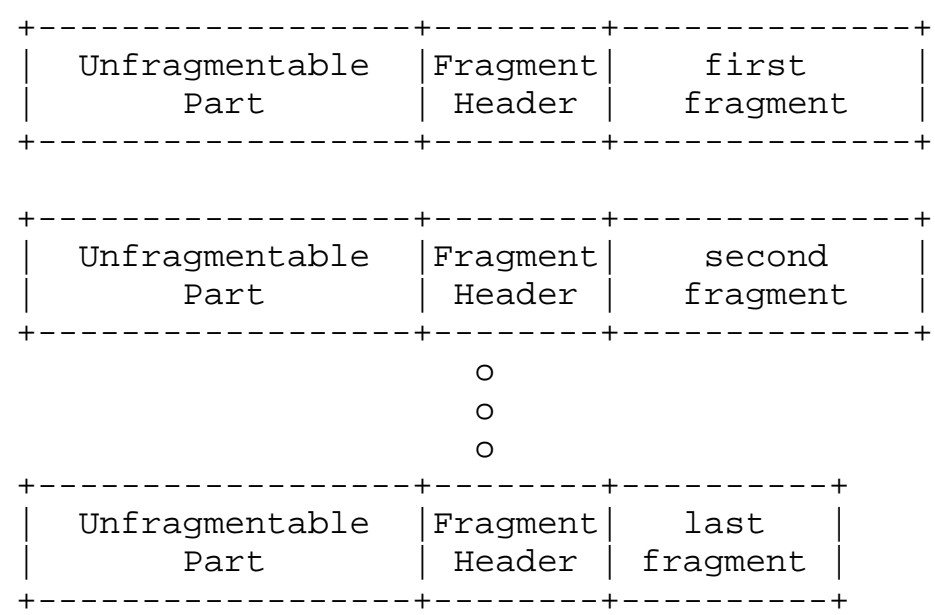

Each fragment packet is composed of:

(1) The Unfragmentable Part of the original packet, with the Payload Length of the original IPv6 header changed to contain the length of this fragment packet only (excluding the length of the IPv6 header itself), and the Next Header field of the last header of the Unfragmentable Part changed to 44.

(2) A Fragment header containing:

The Next Header value that identifies the first header of the Fragmentable Part of the original packet.

A Fragment Offset containing the offset of the fragment, in 8-octet units, relative to the start of the Fragmentable Part of the original packet. The Fragment offset of the first ("leftmost") fragment is 0 .

An $M$ flag value of 0 if the fragment is the last ("rightmost") one, else an M flag value of 1 .

The Identification value generated for the original packet.

(3) The fragment itself.

The lengths of the fragments must be chosen such that the resulting fragment packets fit within the MTU of the path to the packets' destination $(s)$. 
At the destination, fragment packets are reassembled into their original, unfragmented form, as illustrated:

reassembled original packet:

$\left|\begin{array}{c}\text { Unfragmentable } \\ \text { Part }\end{array}\right| \begin{gathered}\text { Pragmentable } \\ \text { Part }\end{gathered}$

The following rules govern reassembly:

An original packet is reassembled only from fragment packets that have the same Source Address, Destination Address, and Fragment Identification.

The Unfragmentable Part of the reassembled packet consists of all headers up to, but not including, the Fragment header of the first fragment packet (that is, the packet whose Fragment offset is zero), with the following two changes:

The Next Header field of the last header of the Unfragmentable Part is obtained from the Next Header field of the first fragment's Fragment header.

The Payload Length of the reassembled packet is computed from the length of the Unfragmentable Part and the length and offset of the last fragment. For example, a formula for computing the Payload Length of the reassembled original packet is:

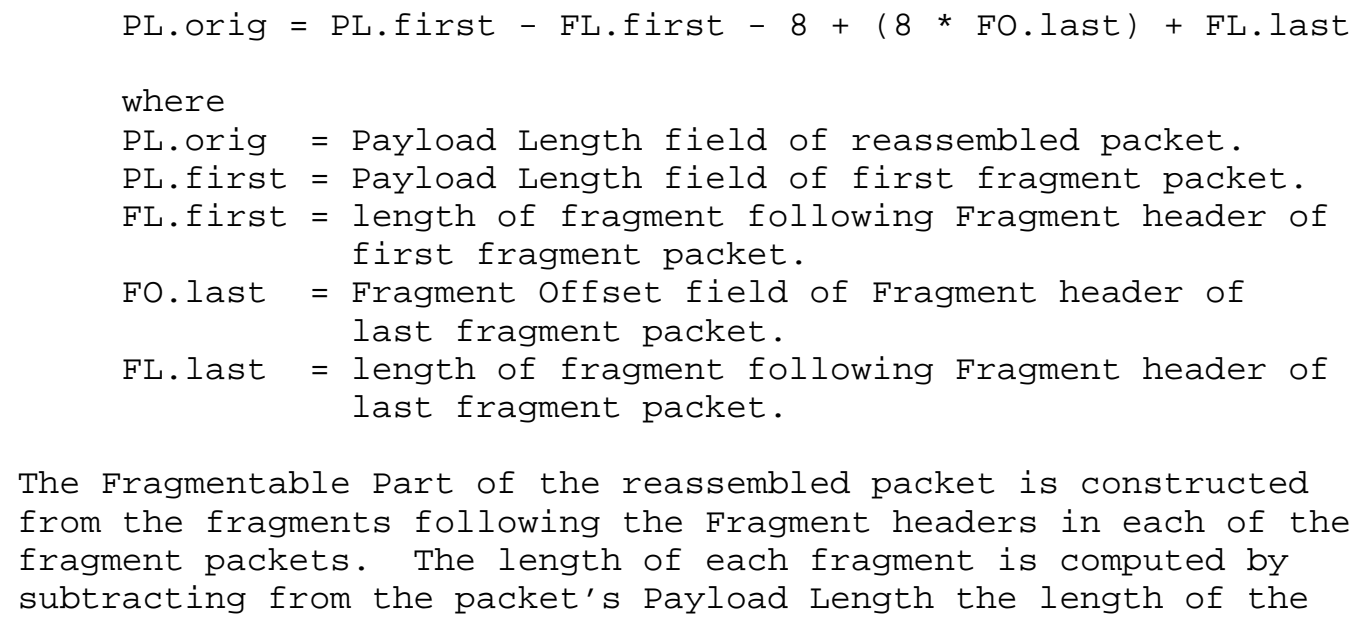

The Fragmentable Part of the reassembled packet is constructed from the fragments following the Fragment headers in each of the fragment packets. The length of each fragment is computed by subtracting from the packet's Payload Length the length of the 
headers between the IPv6 header and fragment itself; its relative position in Fragmentable Part is computed from its Fragment offset value.

The Fragment header is not present in the final, reassembled packet.

The following error conditions may arise when reassembling fragmented packets:

If insufficient fragments are received to complete reassembly of a packet within 60 seconds of the reception of the first-arriving fragment of that packet, reassembly of that packet must be abandoned and all the fragments that have been received for that packet must be discarded. If the first fragment (i.e., the one with a Fragment offset of zerol has been received, an ICMP Time Exceeded -- Fragment Reassembly Time Exceeded message should be sent to the source of that fragment.

If the length of a fragment, as derived from the fragment packet's Payload Length field, is not a multiple of 8 octets and the M flag of that fragment is 1 , then that fragment must be discarded and an ICMP Parameter Problem, Code 0, message should be sent to the source of the fragment, pointing to the Payload Length field of the fragment packet.

If the length and offset of a fragment are such that the Payload Length of the packet reassembled from that fragment would exceed 65,535 octets, then that fragment must be discarded and an ICMP Parameter Problem, Code 0, message should be sent to the source of the fragment, pointing to the Fragment offset field of the fragment packet.

The following conditions are not expected to occur, but are not considered errors if they do:

The number and content of the headers preceding the Fragment header of different fragments of the same original packet may differ. Whatever headers are present, preceding the Fragment header in each fragment packet, are processed when the packets arrive, prior to queueing the fragments for reassembly. Only those headers in the Offset zero fragment packet are retained in the reassembled packet.

The Next Header values in the Fragment headers of different fragments of the same original packet may differ. Only the value from the offset zero fragment packet is used for reassembly. 


\subsection{Destination Options Header}

The Destination Options header is used to carry optional information that need be examined only by a packet's destination node(s). The Destination Options header is identified by a Next Header value of 60 in the immediately preceding header, and has the following format:

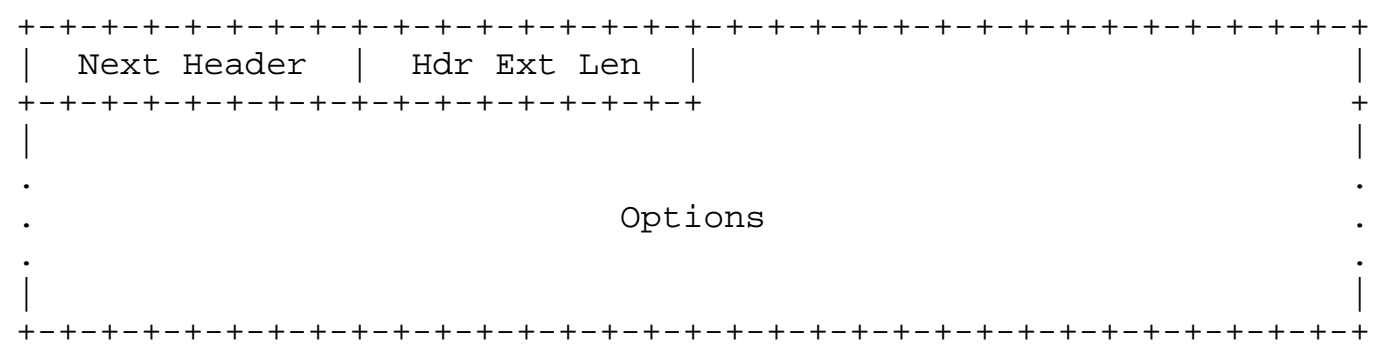

Next Header

8-bit selector. Identifies the type of header immediately following the Destination Options header. Uses the same values as the IPv4 Protocol field [RFC-1700 et seq.].

Hdr Ext Len

8-bit unsigned integer. Length of the Destination Options header in 8-octet units, not including the first 8 octets.

Options

Variable-length field, of length such that the complete Destination Options header is an integer multiple of 8 octets long. Contains one or more TLV-encoded options, as described in section 4.2 .

The only destination options defined in this document are the Pad1 and PadN options specified in section 4.2 .

Note that there are two possible ways to encode optional destination information in an IPv6 packet: either as an option in the Destination Options header, or as a separate extension header. The Fragment header and the Authentication header are examples of the latter approach. Which approach can be used depends on what action is desired of a destination node that does not understand the optional information:

- If the desired action is for the destination node to discard the packet and, only if the packet's Destination Address is not a multicast address, send an ICMP Unrecognized Type message to the packet's Source Address, then the information may be encoded either as a separate header or as an option in the 
Destination Options header whose Option Type has the value 11 in its highest-order two bits. The choice may depend on such factors as which takes fewer octets, or which yields better alignment or more efficient parsing.

- If any other action is desired, the information must be encoded as an option in the Destination Options header whose option Type has the value 00, 01, or 10 in its highest-order two bits, specifying the desired action (see section 4.2).

4.7 No Next Header

The value 59 in the Next Header field of an IPv6 header or any extension header indicates that there is nothing following that header. If the Payload Length field of the IPv6 header indicates the presence of octets past the end of a header whose Next Header field contains 59, those octets must be ignored, and passed on unchanged if the packet is forwarded.

5. Packet Size Issues

IPv6 requires that every link in the internet have an MTU of 1280 octets or greater. On any link that cannot convey a 1280-octet packet in one piece, link-specific fragmentation and reassembly must be provided at a layer below IPv6.

Links that have a configurable MTU (for example, PPP links [RFC1661]) must be configured to have an MTU of at least 1280 octets; it is recommended that they be configured with an MTU of 1500 octets or greater, to accommodate possible encapsulations (i.e., tunneling) without incurring IPv6-layer fragmentation.

From each link to which a node is directly attached, the node must be able to accept packets as large as that link's MTU.

It is strongly recommended that IPv6 nodes implement Path MTU Discovery [RFC-1981], in order to discover and take advantage of path MTUs greater than 1280 octets. However, a minimal IPv6 implementation (e.g., in a boot ROM) may simply restrict itself to sending packets no larger than 1280 octets, and omit implementation of Path MTU Discovery.

In order to send a packet larger than a path's MTU, a node may use the IPv6 Fragment header to fragment the packet at the source and have it reassembled at the destination(s). However, the use of such fragmentation is discouraged in any application that is able to adjust its packets to fit the measured path MTU (i.e., down to 1280 octets). 
A node must be able to accept a fragmented packet that, after reassembly, is as large as 1500 octets. A node is permitted to accept fragmented packets that reassemble to more than 1500 octets. An upper-layer protocol or application that depends on IPv6 fragmentation to send packets larger than the MTU of a path should not send packets larger than 1500 octets unless it has assurance that the destination is capable of reassembling packets of that larger size.

In response to an IPv6 packet that is sent to an IPv4 destination (i.e., a packet that undergoes translation from IPv6 to IPv4), the originating IPv6 node may receive an ICMP Packet Too Big message reporting a Next-Hop MTU less than 1280. In that case, the IPv6 node is not required to reduce the size of subsequent packets to less than 1280, but must include a Fragment header in those packets so that the IPv6-to-IPv4 translating router can obtain a suitable Identification value to use in resulting IPv4 fragments. Note that this means the payload may have to be reduced to 1232 octets (1280 minus 40 for the IPv6 header and 8 for the Fragment header), and smaller still if additional extension headers are used.

6. Flow Labels

The 20-bit Flow Label field in the IPv6 header may be used by a source to label sequences of packets for which it requests special handling by the IPv6 routers, such as non-default quality of service or "real-time" service. This aspect of IPv6 is, at the time of writing, still experimental and subject to change as the requirements for flow support in the Internet become clearer. Hosts or routers that do not support the functions of the Flow Label field are required to set the field to zero when originating a packet, pass the field on unchanged when forwarding a packet, and ignore the field when receiving a packet.

Appendix A describes the current intended semantics and usage of the Flow Label field.

7. Traffic Classes

The 8-bit Traffic Class field in the IPv6 header is available for use by originating nodes and/or forwarding routers to identify and distinguish between different classes or priorities of IPv6 packets. At the point in time at which this specification is being written, there are a number of experiments underway in the use of the IPV4 Type of Service and/or Precedence bits to provide various forms of "differentiated service" for IP packets, other than through the use of explicit flow set-up. The Traffic class field in the IPv6 header is intended to allow similar functionality to be supported in IPv6. 
It is hoped that those experiments will eventually lead to agreement on what sorts of traffic classifications are most useful for IP packets. Detailed definitions of the syntax and semantics of all or some of the IPv6 Traffic Class bits, whether experimental or intended for eventual standardization, are to be provided in separate documents.

The following general requirements apply to the Traffic Class field:

- The service interface to the IPv6 service within a node must provide a means for an upper-layer protocol to supply the value of the Traffic Class bits in packets originated by that upperlayer protocol. The default value must be zero for all 8 bits.

- Nodes that support a specific (experimental or eventual standard) use of some or all of the Traffic class bits are permitted to change the value of those bits in packets that they originate, forward, or receive, as required for that specific use. Nodes should ignore and leave unchanged any bits of the Traffic class field for which they do not support a specific use.

- An upper-layer protocol must not assume that the value of the Traffic Class bits in a received packet are the same as the value sent by the packet's source. 
8. Upper-Layer Protocol Issues

\subsection{Upper-Layer Checksums}

Any transport or other upper-layer protocol that includes the addresses from the IP header in its checksum computation must be modified for use over IPv6, to include the 128-bit IPv6 addresses instead of 32-bit IPV4 addresses. In particular, the following illustration shows the TCP and UDP "pseudo-header" for IPv6:

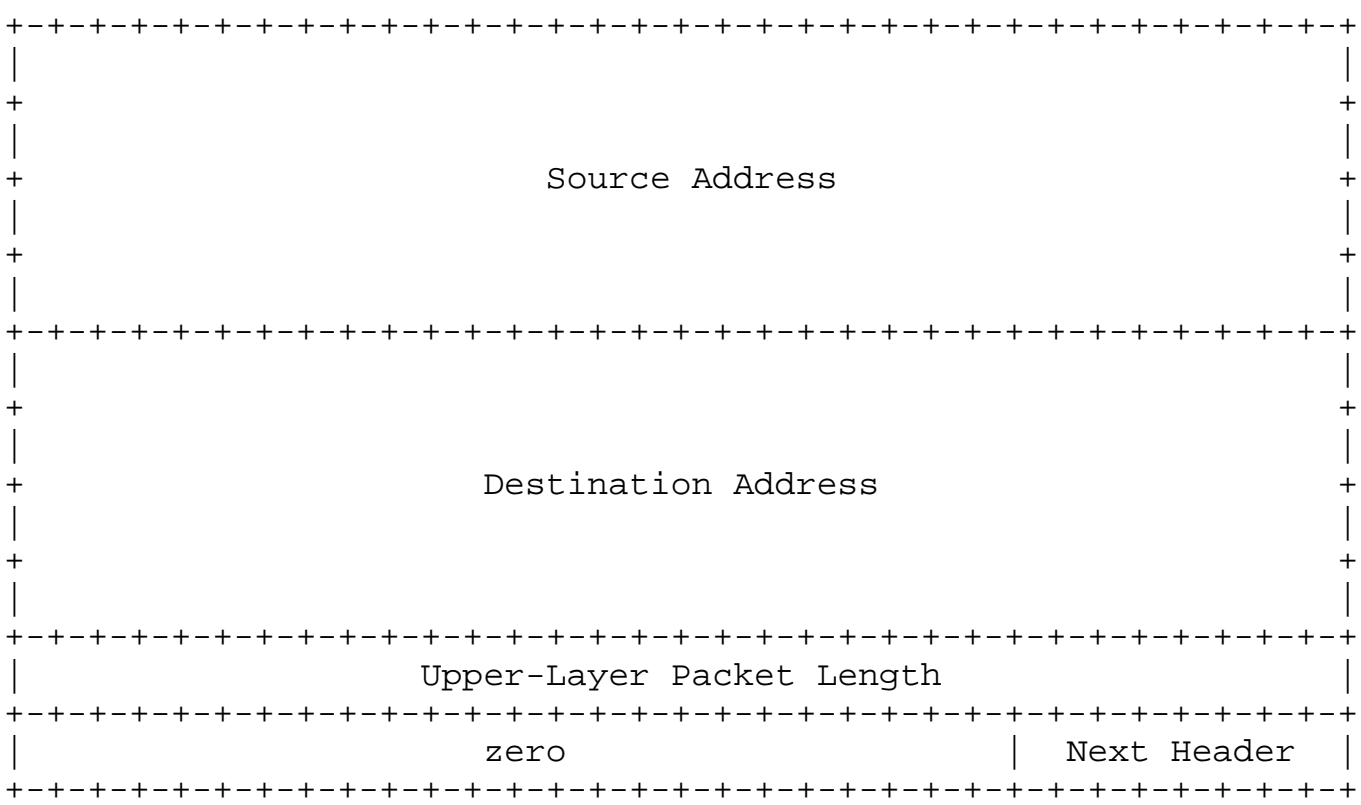

- If the IPv6 packet contains a Routing header, the Destination Address used in the pseudo-header is that of the final destination. At the originating node, that address will be in the last element of the Routing header; at the recipient(s), that address will be in the Destination Address field of the IPv6 header.

- The Next Header value in the pseudo-header identifies the upper-layer protocol (e.g., 6 for TCP, or 17 for UDP). It will differ from the Next Header value in the IPv6 header if there are extension headers between the IPv6 header and the upperlayer header.

- The Upper-Layer Packet Length in the pseudo-header is the length of the upper-layer header and data (e.g., TCP header plus TCP data). Some upper-layer protocols carry their own 
length information (e.g., the Length field in the UDP header); for such protocols, that is the length used in the pseudoheader. Other protocols (such as TCP) do not carry their own length information, in which case the length used in the pseudo-header is the Payload Length from the IPv6 header, minus the length of any extension headers present between the IPv6 header and the upper-layer header.

- Unlike IPv4, when UDP packets are originated by an IPv6 node, the UDP checksum is not optional. That is, whenever originating a UDP packet, an IPV6 node must compute a UDP checksum over the packet and the pseudo-header, and, if that computation yields a result of zero, it must be changed to hex FFFF for placement in the UDP header. IPV6 receivers must discard UDP packets containing a zero checksum, and should log the error.

The IPv6 version of ICMP [ICMPV6] includes the above pseudo-header in its checksum computation; this is a change from the IPv4 version of ICMP, which does not include a pseudo-header in its checksum. The reason for the change is to protect ICMP from misdelivery or corruption of those fields of the IPv6 header on which it depends, which, unlike IPv4, are not covered by an internet-layer checksum. The Next Header field in the pseudo-header for ICMP contains the value 58, which identifies the IPv6 version of ICMP.

8.2 Maximum Packet Lifetime

Unlike IPV4, IPv6 nodes are not required to enforce maximum packet lifetime. That is the reason the IPv4 "Time to Live" field was renamed "Hop Limit" in IPv6. In practice, very few, if any, IPv4 implementations conform to the requirement that they limit packet lifetime, so this is not a change in practice. Any upper-layer protocol that relies on the internet layer (whether IPv4 or IPv6) to limit packet lifetime ought to be upgraded to provide its own mechanisms for detecting and discarding obsolete packets.

\subsection{Maximum Upper-Layer Payload Size}

When computing the maximum payload size available for upper-layer data, an upper-layer protocol must take into account the larger size of the IPv6 header relative to the IPv4 header. For example, in IPV4, TCP's MSS option is computed as the maximum packet size (a default value or a value learned through Path MTU Discovery) minus 40 octets (20 octets for the minimum-length IPv4 header and 20 octets for the minimum-length TCP header). When using TCP over IPv6, the MSS must be computed as the maximum packet size minus 60 octets, 
because the minimum-length IPv6 header (i.e., an IPv6 header with no extension headers) is 20 octets longer than a minimum-length IPv4 header.

\subsection{Responding to Packets Carrying Routing Headers}

When an upper-layer protocol sends one or more packets in response to a received packet that included a Routing header, the response packet(s) must not include a Routing header that was automatically derived by "reversing" the received Routing header UNLESS the integrity and authenticity of the received Source Address and Routing header have been verified (e.g., via the use of an Authentication header in the received packet). In other words, only the following kinds of packets are permitted in response to a received packet bearing a Routing header:

- Response packets that do not carry Routing headers.

- Response packets that carry Routing headers that were NOT derived by reversing the Routing header of the received packet (for example, a Routing header supplied by local configuration).

- Response packets that carry Routing headers that were derived by reversing the Routing header of the received packet IF AND ONLY IF the integrity and authenticity of the source Address and Routing header from the received packet have been verified by the responder. 
Appendix A. Semantics and Usage of the Flow Label Field

A flow is a sequence of packets sent from a particular source to a particular (unicast or multicast) destination for which the source desires special handling by the intervening routers. The nature of that special handling might be conveyed to the routers by a control protocol, such as a resource reservation protocol, or by information within the flow's packets themselves, e.g., in a hop-by-hop option. The details of such control protocols or options are beyond the scope of this document.

There may be multiple active flows from a source to a destination, as well as traffic that is not associated with any flow. A flow is uniquely identified by the combination of a source address and a non-zero flow label. Packets that do not belong to a flow carry a flow label of zero.

A flow label is assigned to a flow by the flow's source node. New flow labels must be chosen (pseudo-) randomly and uniformly from the range 1 to FFFFF hex. The purpose of the random allocation is to make any set of bits within the Flow Label field suitable for use as a hash key by routers, for looking up the state associated with the flow.

All packets belonging to the same flow must be sent with the same source address, destination address, and flow label. If any of those packets includes a Hop-by-Hop Options header, then they all must be originated with the same Hop-by-Hop options header contents (excluding the Next Header field of the Hop-by-Hop Options header). If any of those packets includes a Routing header, then they all must be originated with the same contents in all extension headers up to and including the Routing header (excluding the Next Header field in the Routing header). The routers or destinations are permitted, but not required, to verify that these conditions are satisfied. If a violation is detected, it should be reported to the source by an ICMP Parameter Problem message, Code 0, pointing to the high-order octet of the Flow Label field (i.e., offset 1 within the IPv6 packet).

The maximum lifetime of any flow-handling state established along a flow's path must be specified as part of the description of the state-establishment mechanism, e.g., the resource reservation protocol or the flow-setup hop-by-hop option. A source must not reuse a flow label for a new flow within the maximum lifetime of any flow-handling state that might have been established for the prior use of that flow label. 
When a node stops and restarts (e.g., as a result of a "crash"), it must be careful not to use a flow label that it might have used for an earlier flow whose lifetime may not have expired yet. This may be accomplished by recording flow label usage on stable storage so that it can be remembered across crashes, or by refraining from using any flow labels until the maximum lifetime of any possible previously established flows has expired. If the minimum time for rebooting the node is known, that time can be deducted from the necessary waiting period before starting to allocate flow labels.

There is no requirement that all, or even most, packets belong to flows, i.e., carry non-zero flow labels. This observation is placed here to remind protocol designers and implementors not to assume otherwise. For example, it would be unwise to design a router whose performance would be adequate only if most packets belonged to flows, or to design a header compression scheme that only worked on packets that belonged to flows. 
Appendix B. Formatting Guidelines for Options

This appendix gives some advice on how to lay out the fields when designing new options to be used in the Hop-by-Hop Options header or the Destination Options header, as described in section 4.2. These guidelines are based on the following assumptions:

- One desirable feature is that any multi-octet fields within the Option Data area of an option be aligned on their natural boundaries, i.e., fields of width $n$ octets should be placed at an integer multiple of $n$ octets from the start of the Hop-byHop or Destination Options header, for $\mathrm{n}=1,2,4$, or 8 .

- Another desirable feature is that the Hop-by-Hop or Destination Options header take up as little space as possible, subject to the requirement that the header be an integer multiple of 8 octets long.

- It may be assumed that, when either of the option-bearing headers are present, they carry a very small number of options, usually only one.

These assumptions suggest the following approach to laying out the fields of an option: order the fields from smallest to largest, with no interior padding, then derive the alignment requirement for the entire option based on the alignment requirement of the largest field (up to a maximum alignment of 8 octets). This approach is illustrated in the following examples:

Example 1

If an option $\mathrm{X}$ required two data fields, one of length 8 octets and one of length 4 octets, it would be laid out as follows:

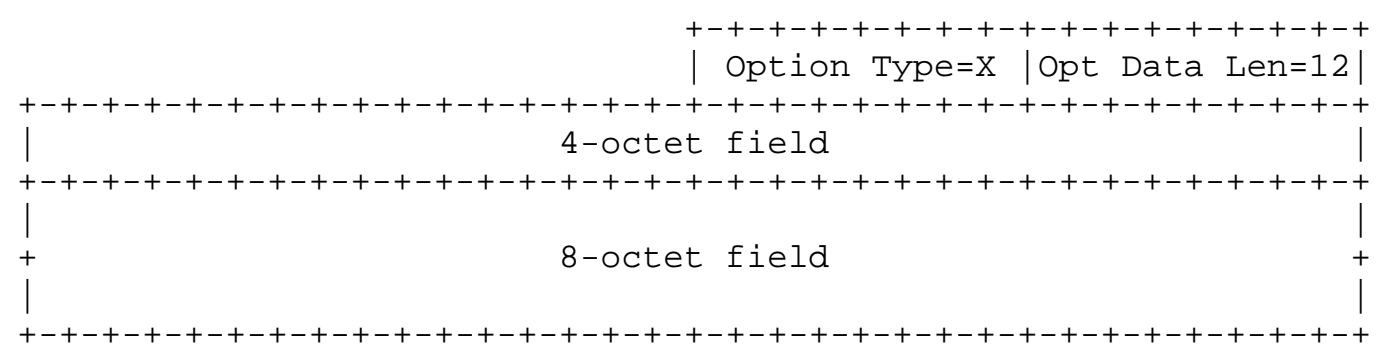


Its alignment requirement is $8 n+2$, to ensure that the 8 -octet field starts at a multiple-of-8 offset from the start of the enclosing header. A complete Hop-by-Hop or Destination Options header containing this one option would look as follows:

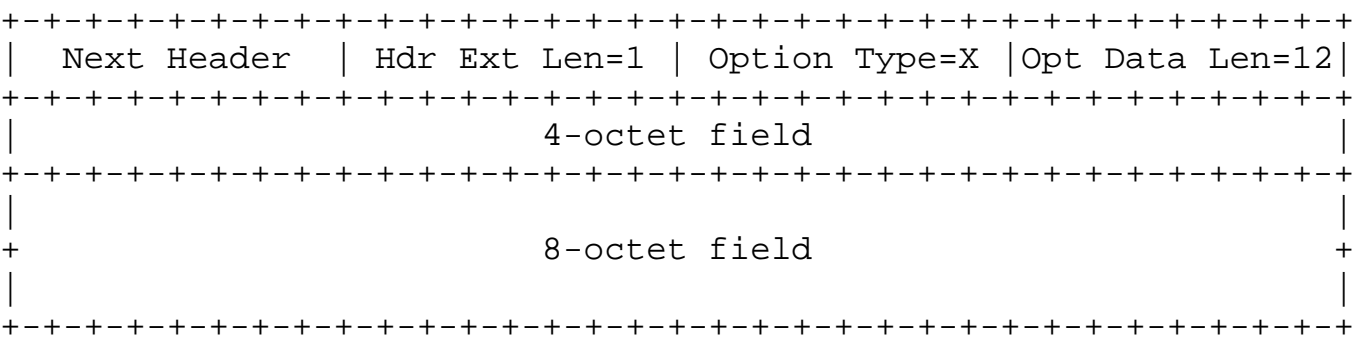

Example 2

If an option $Y$ required three data fields, one of length 4 octets, one of length 2 octets, and one of length 1 octet, it would be laid out as follows:

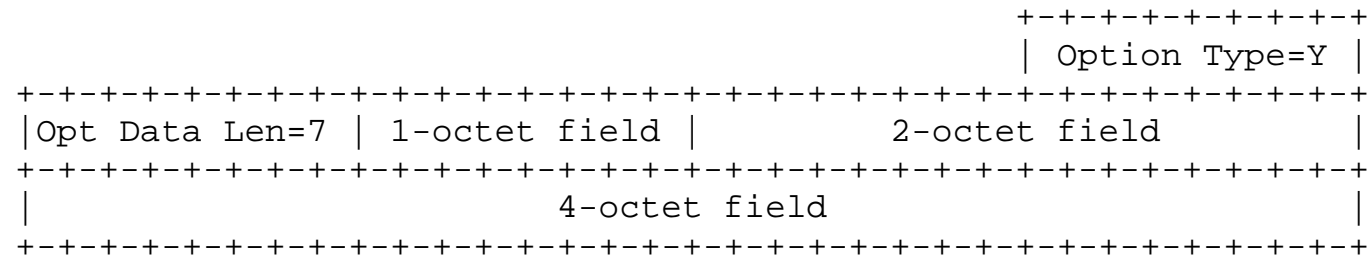

Its alignment requirement is $4 n+3$, to ensure that the 4-octet field starts at a multiple-of-4 offset from the start of the enclosing header. A complete Hop-by-Hop or Destination Options header containing this one option would look as follows:

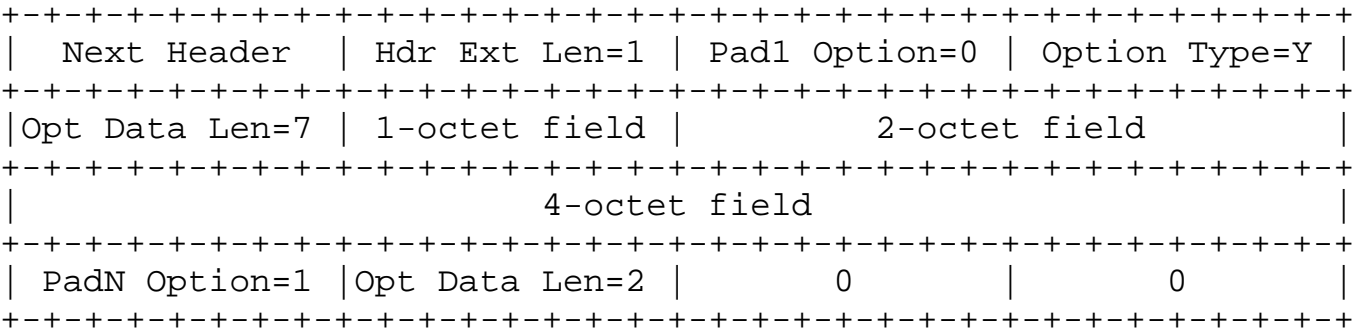


Example 3

A Hop-by-Hop or Destination Options header containing both options $\mathrm{X}$ and $Y$ from Examples 1 and 2 would have one of the two following formats, depending on which option appeared first:
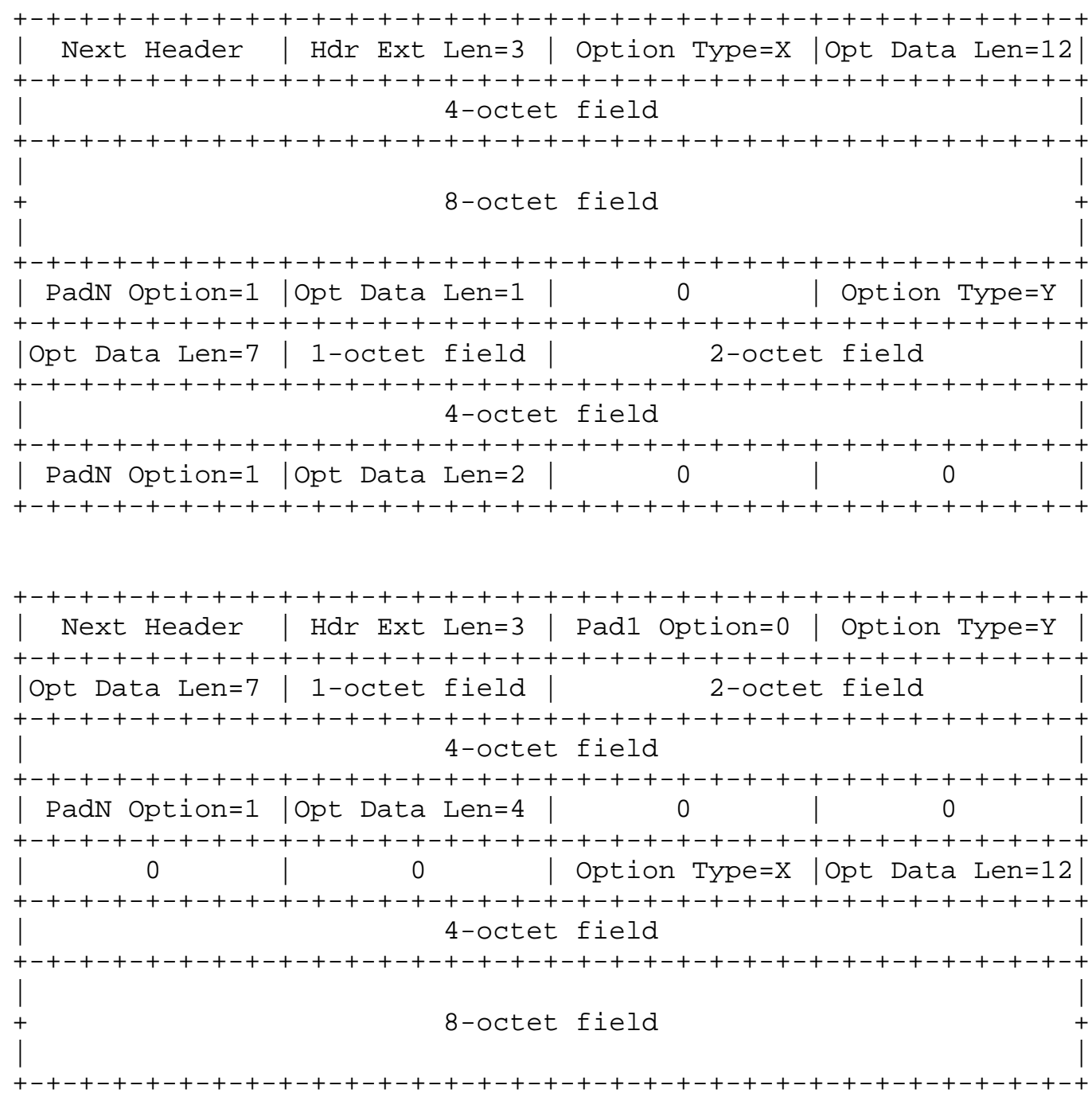


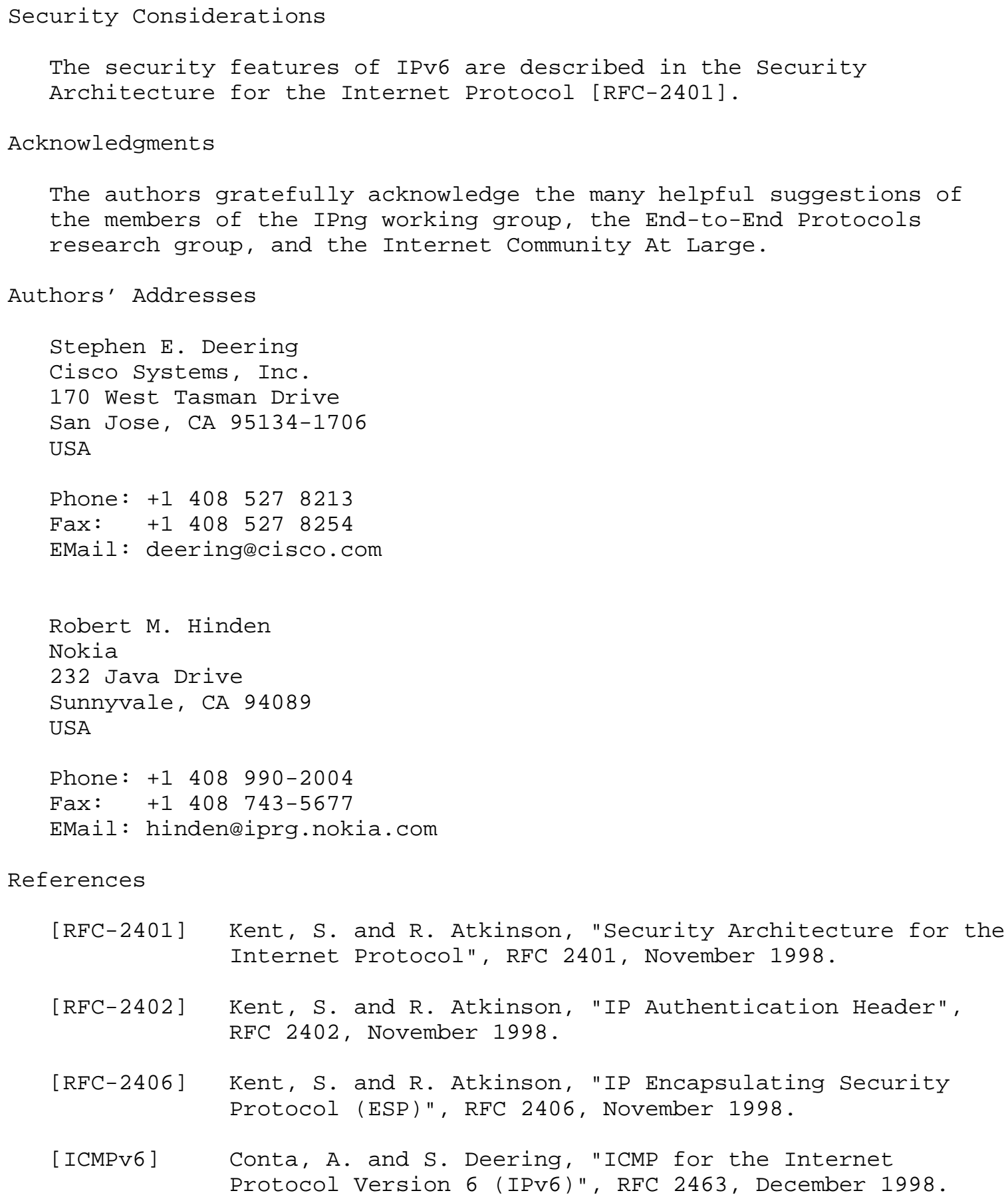


[ADDRARCH] Hinden, R. and S. Deering, "IP Version 6 Addressing Architecture", RFC 2373, July 1998.

[RFC-1981] McCann, J., Mogul, J. and S. Deering, "Path MTU Discovery for IP version 6", RFC 1981, August 1996.

[RFC-791] Postel, J., "Internet Protocol", STD 5, RFC 791, September 1981 .

[RFC-1700] Reynolds, J. and J. Postel, "Assigned Numbers", STD 2, RFC 1700, October 1994. See also: http://www.iana.org/numbers.html

[RFC-1661] Simpson, W., "The Point-to-Point Protocol (PPP)", STD 51, RFC 1661, July 1994.

CHANGES SINCE RFC-1883

This memo has the following changes from RFC-1883. Numbers identify the Internet-Draft version in which the change was made.

02) Removed all references to jumbograms and the Jumbo Payload option (moved to a separate document).

02) Moved most of Flow Label description from section 6 to (new) Appendix A.

02) In Flow Label description, now in Appendix A, corrected maximum Flow Label value from FFFFFF to FFFFF (i.e., one less "F") due to reduction of size of Flow Label field from 24 bits to 20 bits.

02) Renumbered (relettered?) the previous Appendix A to be Appendix B.

02) Changed the wording of the Security Considerations section to avoid dependency loop between this spec and the IPsec specs.

02) Updated R. Hinden's email address and company affiliation.

01) In section 3, changed field name "Class" to "Traffic Class" and increased its size from 4 to 8 bits. Decreased size of Flow Label field from 24 to 20 bits to compensate for increase in Traffic Class field. 
01) In section 4.1, restored the order of the Authentication Header and the ESP header, which were mistakenly swapped in the 00 version of this memo.

01) In section 4.4, deleted the Strict/Loose Bit Map field and the strict routing functionality from the Type 0 Routing header, and removed the restriction on number of addresses that may be carried in the Type 0 Routing header (was limited to 23 addresses, because of the size of the strict/loose bit map).

01) In section 5, changed the minimum IPv6 MTU from 576 to 1280 octets, and added a recommendation that links with configurable MTU (e.g., PPP links) be configured to have an MTU of at least 1500 octets.

01) In section 5, deleted the requirement that a node must not send fragmented packets that reassemble to more than 1500 octets without knowledge of the destination reassembly buffer size, and replaced it with a recommendation that upper-layer protocols or applications should not do that.

01) Replaced reference to the IPv4 Path MTU Discovery spec (RFC1191) with reference to the IPv6 Path MTU Discovery spec (RFC1981), and deleted the Notes at the end of section 5 regarding Path MTU Discovery, since those details are now covered by RFC1981.

01) In section 6, deleted specification of "opportunistic" flow set-up, and removed all references to the 6-second maximum lifetime for opportunistically established flow state.

01) In section 7, deleted the provisional description of the internal structure and semantics of the Traffic Class field, and specified that such descriptions be provided in separate documents.

00) In section 4, corrected the Code value to indicate "unrecognized Next Header type encountered" in an ICMP Parameter Problem message (changed from 2 to 1 ).

00) In the description of the Payload Length field in section 3 , and of the Jumbo Payload Length field in section 4.3, made it clearer that extension headers are included in the payload length count. 
00) In section 4.1, swapped the order of the Authentication header and the ESP header. (NOTE: this was a mistake, and the change was undone in version 01.)

00) In section 4.2, made it clearer that options are identified by the full 8-bit option Type, not by the low-order 5 bits of an Option Type. Also specified that the same Option Type numbering space is used for both Hop-by-Hop Options and Destination Options headers.

00) In section 4.4, added a sentence requiring that nodes processing a Routing header must send an ICMP Packet Too Big message in response to a packet that is too big to fit in the next hop link (rather than, say, performing fragmentation).

00) Changed the name of the IPv6 Priority field to "Class", and replaced the previous description of Priority in section 7 with a description of the class field. Also, excluded this field from the set of fields that must remain the same for all packets in the same flow, as specified in section 6 .

00) In the pseudo-header in section 8.1, changed the name of the "Payload Length" field to "Upper-Layer Packet Length". Also clarified that, in the case of protocols that carry their own length info (like non-jumbogram UDP), it is the upper-layerderived length, not the IP-layer-derived length, that is used in the pseudo-header.

00) Added section 8.4, specifying that upper-layer protocols, when responding to a received packet that carried a Routing header, must not include the reverse of the Routing header in the response packet(s) unless the received Routing header was authenticated.

00) Fixed some typos and grammatical errors.

00) Authors' contact info updated. 
Full Copyright statement

Copyright (C) The Internet Society (1998). All Rights Reserved.

This document and translations of it may be copied and furnished to others, and derivative works that comment on or otherwise explain it or assist in its implementation may be prepared, copied, published and distributed, in whole or in part, without restriction of any kind, provided that the above copyright notice and this paragraph are included on all such copies and derivative works. However, this document itself may not be modified in any way, such as by removing the copyright notice or references to the Internet society or other Internet organizations, except as needed for the purpose of developing Internet standards in which case the procedures for copyrights defined in the Internet Standards process must be followed, or as required to translate it into languages other than English.

The limited permissions granted above are perpetual and will not be revoked by the Internet society or its successors or assigns.

This document and the information contained herein is provided on an "AS IS" basis and THE INTERNET SOCIETY AND THE INTERNET ENGINEERING TASK FORCE DISCLAIMS ALL WARRANTIES, EXPRESS OR IMPLIED, INCLUDING BUT NOT LIMITED TO ANY WARRANTY THAT THE USE OF THE INFORMATION HEREIN WILL NOT INFRINGE ANY RIGHTS OR ANY IMPLIED WARRANTIES OF MERCHANTABILITY OR FITNESS FOR A PARTICULAR PURPOSE. 